\title{
CAApp: Um Aplicativo Móvel de Comunicação Aumentativa e Alternativa para Pessoas com Deficiência Motora Severa
}

\author{
Yves L. Coelho ${ }^{1}$, Alexandre L. C. Bissoli ${ }^{1}$, Mariana M. Sime ${ }^{1}$, Teodiano F. Bastos \\ Filho $^{1}$ \\ ${ }^{1}$ Laboratório de Automação Inteligente - Programa de Pós-Graduação em Engenharia \\ Elétrica - Universidade Federal do Espírito Santo (UFES) \\ Av. Fernando Ferrari, 514, Goiabeiras, 29075-910 - Vitória-ES \\ yvesluduvico@gmail.com, alexandre-bissoli@hotmail.com, \\ mariana.midori@gmail.com, teodiano.bastos@ufes.br
}

\begin{abstract}
This work presents an Augmentative and Alternative Communication system, using a mobile application, to offer a communication means for people with severe motor disabilities. The application was developed for the Android platform and integrated to a smart environment system. The present paper approaches the alternative means of communication benefits for individuals with specific needs, the developed application features and the system operation process.
\end{abstract}

Resumo. Este trabalho apresenta um sistema de Comunicação Aumentativa e Alternativa, utilizando um aplicativo móvel, para oferecer um meio de comunicação a pessoas com deficiências motoras severas. A aplicação foi desenvolvida para a plataforma Android e integrada a um sistema de ambiente inteligente. O presente artigo aborda os beneficios de um meio alternativo de comunicação para indivíduos com necessidades específicas, as funcionalidades da aplicação desenvolvida e o processo de operação do sistema.

\section{Introdução}

Pessoas com deficiências motoras severas, tais como paralisia cerebral, distrofias musculares, esclerose múltipla, esclerose lateral amiotrófica, acidente vascular cerebral entre outras, podem apresentar déficit na comunicação, comprometendo a interação com seus familiares ou cuidadores [Pedretti e Early 2004]. A Comunicação Aumentativa e Alternativa (CAA) compensa a dificuldade de comunicação destes indivíduos, utilizando um meio alternativo de comunicação ou ampliando suas habilidades de comunicação. A CAA utiliza gestos, língua de sinais, expressões faciais, pranchas de alfabeto ou de símbolos pictográficos e sistemas sofisticados de computador com voz sintetizada. Além de contribuir para a socialização, a CAA permite que uma pessoa com dificuldades de comunicação possa informar problemas a familiares ou cuidadores, fazer pedidos e escolhas relacionados ao cotidiano e pedir auxílio [Glennen 1997] [Buning 2013].

De acordo com a USSAAC (2015), os dispositivos de CAA podem ser classificados em dois grupos: dispositivos de baixa tecnologia e de alta tecnologia. Os dispositivos de baixa tecnologia não precisam de baterias, eletricidade ou componentes 
eletrônicos. Alguns exemplos são pranchas, tabuleiros e livros com letras, palavras, frases ou símbolos que são indicados pelo usuário de diversas formas. Por outro lado, os dispositivos de alta tecnologia são dispositivos eletrônicos que permitem armazenamento e recuperação de mensagens, muitas vezes utilizando saída de voz. Podem ser dispositivos dedicados para CAA ou computadores adaptados. Podem ter tela estática, com todas as opções possíveis apresentadas, ou tela dinâmica, apresentando apenas um grupo de opções por vez. A seleção de opções pode ser direta, com acesso a uma tela ou a um teclado com parte do corpo, mouses ou joysticks, ou indireta, quando é realizada uma varredura entre as opções e o usuário deve intervir na alternativa desejada.

O exponencial crescimento do uso de smartphones contribui para o desenvolvimento de aplicativos móveis com diversos propósitos, entre eles aqueles que facilitam atividades do cotidiano. Apesar das dificuldades encontradas para uso de smartphones por pessoas com deficiência motora severa [Bryen e Pecunas 2004], estes dispositivos, com aplicativos específicos, podem ser facilitadores de comunicação com outras pessoas. Portanto, os smartphones podem ser utilizados como dispositivos de CAA de alta tecnologia.

Com o objetivo de proporcionar maior independência a pessoas com deficiência motora severa, os pesquisadores do Laboratório de Automação Inteligente da Universidade Federal do Espírito Santo (LAI-UFES) estão desenvolvendo um sistema de ambiente inteligente [Bissoli et al. 2015a] [Bissoli et al. 2015b]. Por meio de sinais de eletromiografia de superfície (sEMG) e de eletro-oculografia (EOG), é possível controlar equipamentos domésticos como ar condicionado, ventilador, televisão, aparelho de som, iluminação etc. $\mathrm{O}$ acionamento ocorre por meio de movimentos voluntários de cerrar os dentes, elevar as sobrancelhas e movimentar horizontalmente os olhos (direita/esquerda), que são capturados pelo dispositivo Emotiv EPOC. Uma interface gráfica, visualizada em um monitor acoplado a uma cadeira de rodas motorizada, apresenta as opções de equipamentos e o usuário pode escolher qual deseja acionar ou desligar.

Este trabalho apresenta um recurso de CAA, adicionado ao sistema descrito anteriormente, que utiliza um aplicativo móvel, chamado CAApp, em um smartphone, com o objetivo de auxiliar a comunicação entre a pessoa com deficiência e seu cuidador. O aplicativo desenvolvido permite que o usuário realize uma ligação telefônica ou envie mensagens de texto (SMS, do inglês Short Message Service) automáticas para seu cuidador por intermédio de sinais de sEMG e EOG utilizando a mesma interface gráfica do sistema de ambiente inteligente.

O presente artigo está estruturado da seguinte forma: a seção seguinte apresenta trabalhos relacionados com este projeto, destacando o desenvolvimento de dispositivos eletrônicos de CAA utilizando sinais biológicos; a Seção 3 apresenta o ambiente inteligente para pessoas com deficiência desenvolvido no LAI-UFES e aborda o aplicativo CAApp implementado neste trabalho; a Seção 4 apresenta os resultados do desenvolvimento do aplicativo, mostrando o processo de operação do sistema de CAA; por fim, a Seção 5 aborda as considerações finais e apresenta algumas propostas de trabalhos futuros. 


\section{Trabalhos Relacionados}

Sistemas eletrônicos de CAA estão presentes na literatura científica, principalmente a partir do final do século passado, e empregam diferentes técnicas de acordo com a tecnologia disponível e com o contexto da aplicação. No ambiente escolar, dispositivos de CAA são desenvolvidos para auxiliar o aprendizado de crianças que apresentam difículdades de comunicação. Alguns exemplos são um sistema de CAA, desenvolvido no início da década de 90, para crianças com paralisia cerebral [Palerm e Ruíz 1992], e um aplicativo de CAA em um pequeno computador para autistas manifestarem seus desejos [Torii et al. 2012].

No contexto de dispositivos de CAA para pessoas com deficiências motoras severas encontram-se cada vez mais pesquisas. No decorrer das últimas três décadas, diversos dispositivos eletrônicos de CAA utilizando mouses, joysticks e botões foram desenvolvidos para atender pessoas com dificuldade de se expressar através da fala. No entanto, desenvolvimentos recentes de estratégias de CAA para pessoas com movimentos mínimos têm sido propostos, entre eles rastreamento do movimento da cabeça e dos olhos, e reconhecimento de gestos e de fala residual [Fager et al. 2012]. Interfaces que utilizam sinais biológicos, como sinais de eletroencefalografia (EEG), sEMG e EOG, são propostas ainda mais recentes e que tornam possível um meio ainda mais eficiente de CAA para pessoas com deficiências motoras severas.

Diversos trabalhos propõem uma interface cérebro-computador para estabelecer a comunicação entre o usuário e um sistema de CAA [Blankertz et al. 2006] [FazelRezai et al. 2012] [Al-Abdullatif et al. 2013]. Entretanto, quando os movimentos de músculos da face são preservados, podem-se utilizar sinais de eletromiografia de superfície (sEMG) para realizar a interação com um dispositivo. Numerosos projetos de interfaces homem-computador utilizando sinais de sEMG e EOG têm sido propostos recentemente [Choi, Rim e Kim 2011] [Banerjee et al. 2012] [Lam 2012] [Paul et al. 2013].

Com o avanço da tecnologia móvel, sistemas de CAA estão sendo desenvolvidos utilizando uma interface entre usuário e dispositivos móveis, como smartphones e tablets. Kim, Park e Ghovanloo (2012) desenvolveram um sistema acionado pelo movimento da língua do usuário para manipular um smartphone, tornando possível o uso de aplicativos do dispositivo por pessoas com deficiência motora severa. Quintela, Mendes e Correia (2013) apresentam um aplicativo móvel de comunicação aumentativa e alternativa voltado principalmente para o processo de inclusão social de pessoas com dificuldades de comunicação.

Nota-se que diferentes tecnologias têm sido empregadas para resolver problemas de comunicação de pessoas com dificuldades de comunicação básica. Muitos destes trabalhos dedicam-se a permitir que o usuário escreva uma mensagem por sinais biológicos, o que ainda é um processo lento. O presente trabalho busca atender a necessidade de comunicação imediata e eficiente entre uma pessoa com deficiência motora severa e seu cuidador, utilizando recursos modernos de dispositivos móveis e de captura de sinais biológicos. 


\section{O Aplicativo de Comunicação Aumentativa e Alternativa}

A pessoa com deficiência é aqui referida como usuário do sistema, enquanto o termo cuidador refere-se ao responsável pela pessoa com deficiência, podendo ser um cuidador formal (contratado para este serviço) ou um cuidador informal (um familiar, um amigo etc). Esta seção aborda o sistema de ambiente inteligente desenvolvido no LAI-UFES e o aplicativo móvel CAApp desenvolvido neste trabalho para oferecer um meio de comunicação aumentativa e alternativa.

\subsection{O Ambiente Inteligente}

No âmbito dos ambientes inteligentes voltados para as tecnologias assistivas, desenvolveu-se no LAI-UFES um sistema de acionamento de equipamentos por rádio frequência (RF) para cadeirantes. Assim, permite-se que uma pessoa com deficiência motora severa ligue e desligue eletrodomésticos sem precisar de ajuda, utilizando sinais de sEMG e EOG [Bissoli et al. 2015a]. Foi projetada e confeccionada uma Caixa Inteligente em acrílico que permite acionar até quatro eletrodomésticos em um ambiente, por exemplo, ventilador, televisão, ar condicionado, lâmpadas, entre outros. $\mathrm{O}$ headset Emotiv EPOC, dispositivo da empresa Emotiv, é utilizado para capturar os sinais biológicos pertinentes (sEMG e EOG).

O usuário, visualizando opções em uma interface gráfica e utilizando o headset Emotiv EPOC, pode enviar comandos por movimentos da face e dos olhos - sinais de sEMG e EOG - que são interpretados no computador de bordo da cadeira de rodas por um software da Emotiv e pelo Matlab. Na sequência, os comandos são enviados para o microcontrolador mestre que está integrado à cadeira. $\mathrm{O}$ microcontrolador então transmite, por rádio frequência, os comandos até a Caixa Inteligente, que possui um receptor RF integrado ao microcontrolador escravo, onde é feito o acionamento do equipamento correspondente [Bissoli et al. 2015b].

No presente trabalho, uma nova opção foi inserida na interface gráfica. O item "celular" oferece a possibilidade do usuário utilizar um smartphone a bordo da cadeira de rodas para se comunicar, de forma alternativa, com seu cuidador. $\mathrm{Na}$ imagem à esquerda na Figura 1 apresenta-se a interface gráfica, desenvolvida no Matlab, com as opções de diferentes ambientes, para acionamento de equipamentos, e a nova opção "celular" inserida.

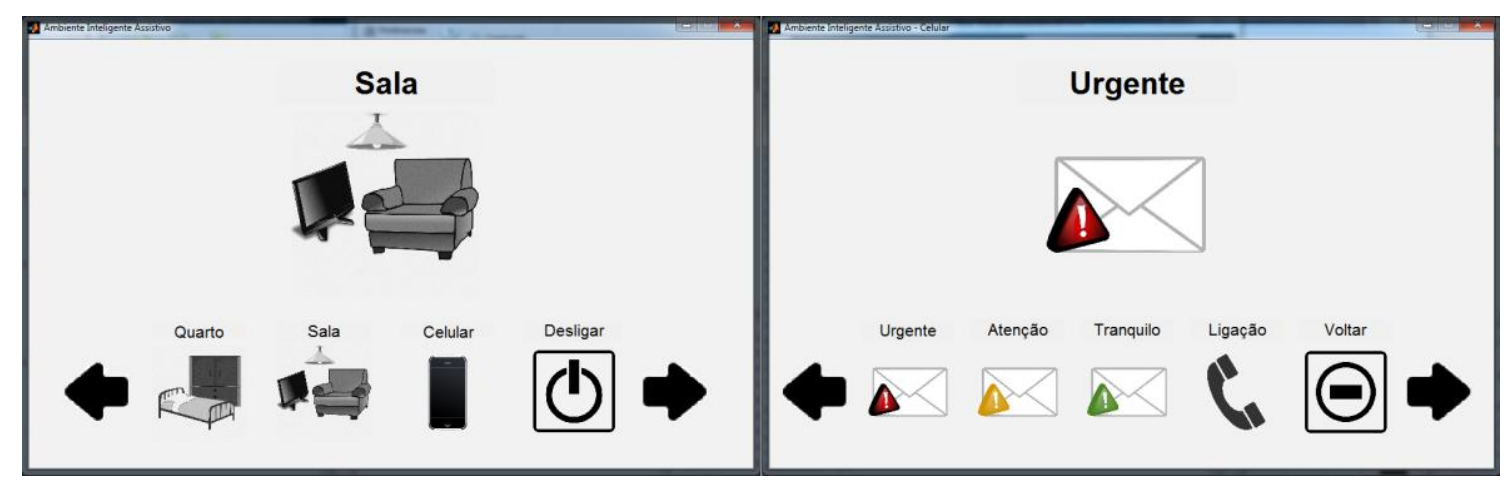

Figura 1. Menu principal da interface gráfica de controle do ambiente inteligente (à esquerda); e submenu com opções disponíveis para interação com o smartphone (à direita) 


\subsection{O Sistema de Comunicação Aumentativa e Alternativa}

Este trabalho apresenta a integração de um meio de CAA ao sistema de ambiente inteligente descrito acima, permitindo que o usuário do sistema entre em contato com seu cuidador utilizando um smartphone. Com este propósito, uma opção foi inserida na interface gráfica referindo-se ao smartphone do usuário. Ao escolher esta opção, são apresentadas outras alternativas: realizar uma ligação ou enviar uma entre três possíveis mensagens de texto automáticas, como pode-se observar na Figura 1. A Figura 2 apresenta o diagrama de comunicação entre os diversos elementos do sistema de comunicação aumentativa e alternativa.

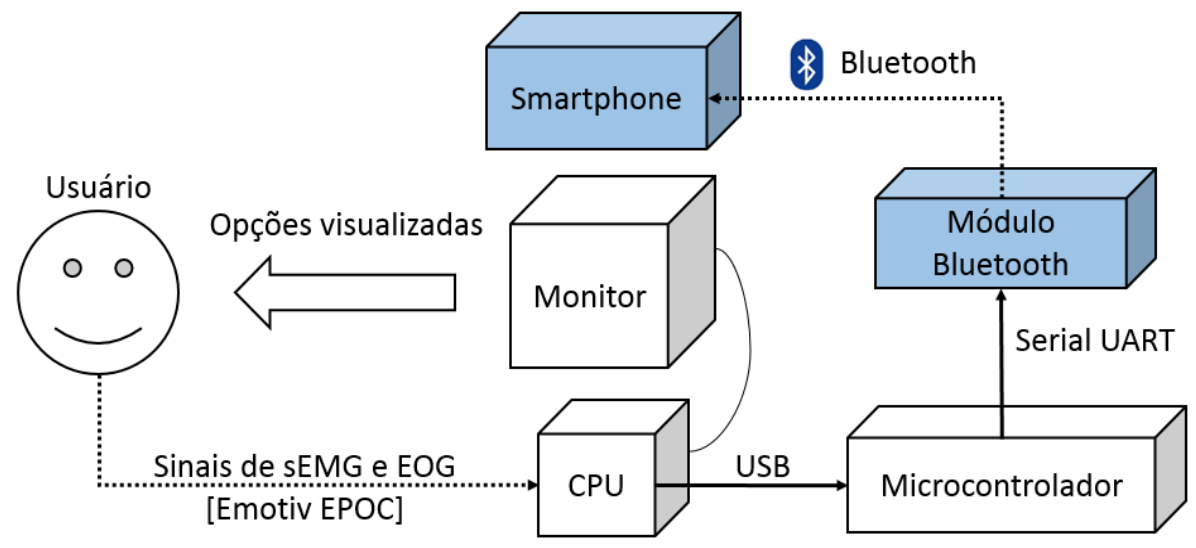

Figura 2. Esquematização do sistema de CAA.

Na Figura 2, setas contínuas indicam comunicação com fio, enquanto setas pontilhadas indicam comunicação sem fio. Os elementos em azul foram adicionados ao sistema principal para prover um meio de comunicação alternativa ao usuário. Um módulo Bluetooth foi integrado ao microcontrolador mestre do sistema para permitir a comunicação sem fio entre este e o smartphone do usuário. O módulo utilizado, chamado HC-05, comunica-se com o microcontrolador através da comunicação serial UART. De acordo com a opção selecionada pelo usuário na interface gráfica utilizando movimentos da face e dos olhos, um determinado comando é enviado ao microcontrolador e encaminhado ao aplicativo CAApp no smartphone através do módulo Bluetooth. Ao receber o comando, o aplicativo realiza uma das possíveis tarefas: ligação telefônica ou mensagem de texto.

\subsection{Descrição do Aplicativo Móvel}

O aplicativo móvel CAApp apresentado neste trabalho foi desenvolvido para o sistema operacional Android, de forma a atender uma grande parcela de smartphones disponíveis no mercado. Além disso, a plataforma Android conta com uma ampla comunidade de desenvolvedores, onde se encontra suporte nos mais diversos tipos de aplicações. O aplicativo desenvolvido foi implementado no ambiente de desenvolvimento Android Studio e o seu digrama completo de funcionamento é apresentado na Figura 3.

Previamente à operação do sistema, o Bluetooth deve ser ligado no smartphone do usuário, e o módulo Bluetooth integrado ao sistema deve ser devidamente pareado. Assim que o aplicativo é executado pelo cuidador, a tela principal apresenta as seguintes 


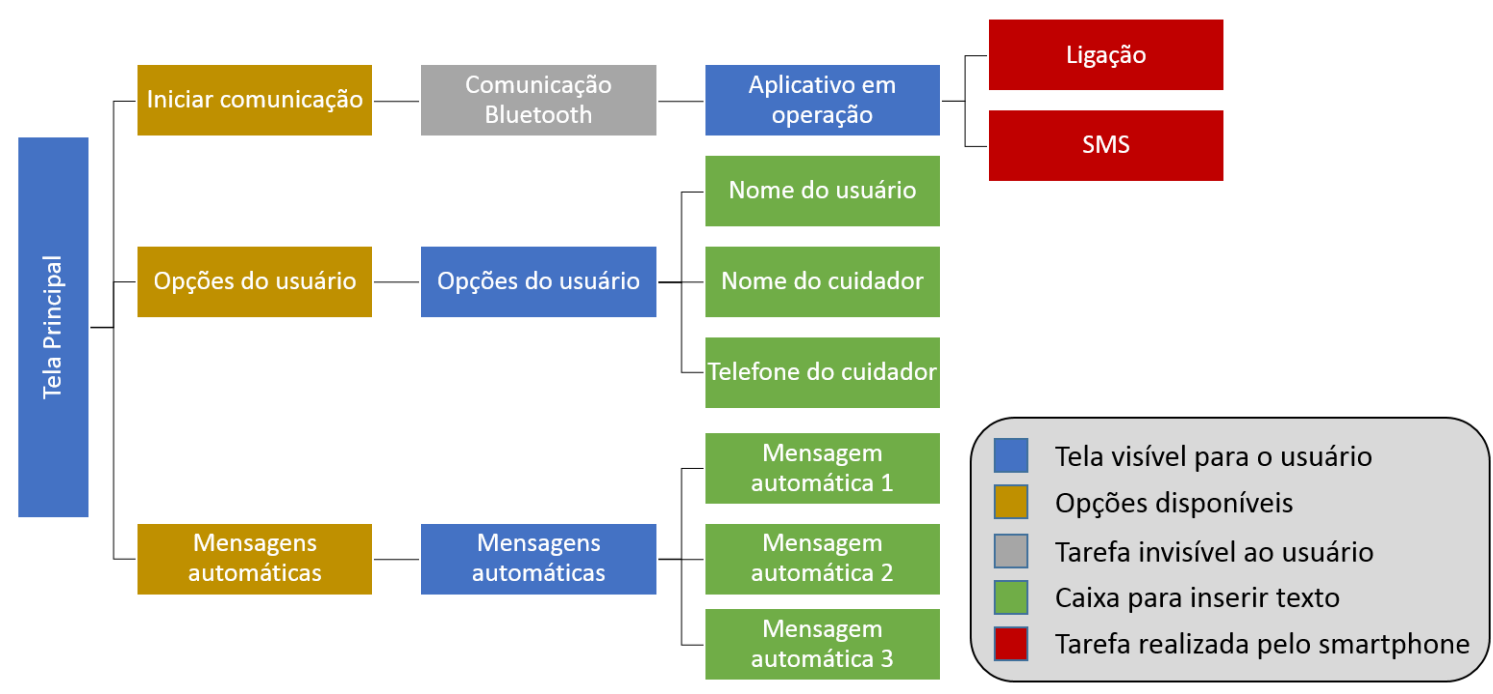

Figura 3. Diagrama completo dos processos do aplicativo desenvolvido

opções: iniciar comunicação, opções do usuário e mensagens automáticas. Ao selecionar a opção "iniciar comunicação", o smartphone conecta-se ao módulo Bluetooth integradoao sistema e executa uma nova tela, na qual permanece aguardando o recebimento de um comando do usuário por meio da interface gráfica do sistema de ambiente inteligente. De acordo com o comando recebido, o smartphone deve executar uma entre as opções abaixo.

- Ligação Telefônica: ao receber o comando referente a esta operação, o aplicativo executa a aplicação nativa de ligação do smartphone do usuário, utilizando o número de telefone do cuidador, previamente inserido no aplicativo; assim que a ligação é iniciada, o viva-voz do dispositivo é ligado para que o usuário possa falar sem a necessidade de manter o smartphone próximo ao seu rosto.

- Mensagens de texto (SMS): caso receba um dos três comandos referentes a mensagens de texto, o aplicativo envia uma das três mensagens automáticas para o número do cuidador previamente cadastrado no aplicativo; cada um dos comandos recebidos está associado a uma das três mensagens de texto que foram inseridas no aplicativo pelo cuidador.

Na tela exibida após a seleção do item "opções do usuário" são apresentadas caixas de textos nas quais o cuidador deve inserir o nome do usuário, o seu próprio nome e o seu número de telefone celular. $\mathrm{O}$ nome do usuário é a assinatura das mensagens de texto. O nome do cuidador é inserido no início da mensagem. Já o número de telefone do cuidador é para o qual a ligação é efetuada e as mensagens são enviadas.

A terceira opção, "mensagens automáticas", executa uma nova tela que contém três caixas de textos correspondentes a cada uma das mensagens de texto automáticas. $\mathrm{O}$ cuidador é o responsável por cadastrar cada umas das mensagens, juntamente com o usuário. Cada mensagem está associada a um símbolo de exclamação de diferente cor, exibido ao lado da caixa de texto, que se refere ao nível de urgência exigida pelo usuário. Os símbolos de exclamação vermelho, amarelo e verde indicam, respectivamente, níveis de urgência alto, médio e baixo. Estes símbolos de exclamação 
também são utilizados nos ícones das mensagens na interface gráfica do sistema principal para que o usuário possa fazer uma associação imediata no momento de enviar o SMS.

\subsection{Desenvolvimento do Aplicativo Móvel}

O Android disponibiliza diversas Interfaces de Programação de Aplicações (API, do inglês Application Programming Interface), divididas em pacotes, ao desenvolvedor, para possibilitar a utilização de funcionalidades do dispositivo móvel por uma aplicação. Neste projeto, foram utilizadas API do Bluetooth, disponíveis no pacote "android.bluetooth", de forma a permitir, neste caso, que a aplicação se comunique com outro dispositivo via Bluetooth e receba dados deste dispositivo. É importante destacar que uma permissão de utilização das funcionalidades do Bluetooth do smartphone deve ser declarada no arquivo pertinente, chamado AndroidManifest.

Para enviar mensagens de texto por meio da aplicação utiliza-se a classe smsManager, disponível no pacote "android.telephony". Já para realizar uma ligação telefônica deve ser chamada uma ação para utilizar a aplicação nativa de ligação do dispositivo móvel. Tanto para SMS, como para ligação, deve-se declarar permissão de uso das devidas funcionalidades do smartphone no arquivo AndroidManifest.

\section{Resultados}

Esta seção apresenta o funcionamento do aplicativo móvel CAApp desenvolvido neste trabalho e descrito na subseção 3.3. A Figura 4 apresenta as telas de configurações do aplicativo.

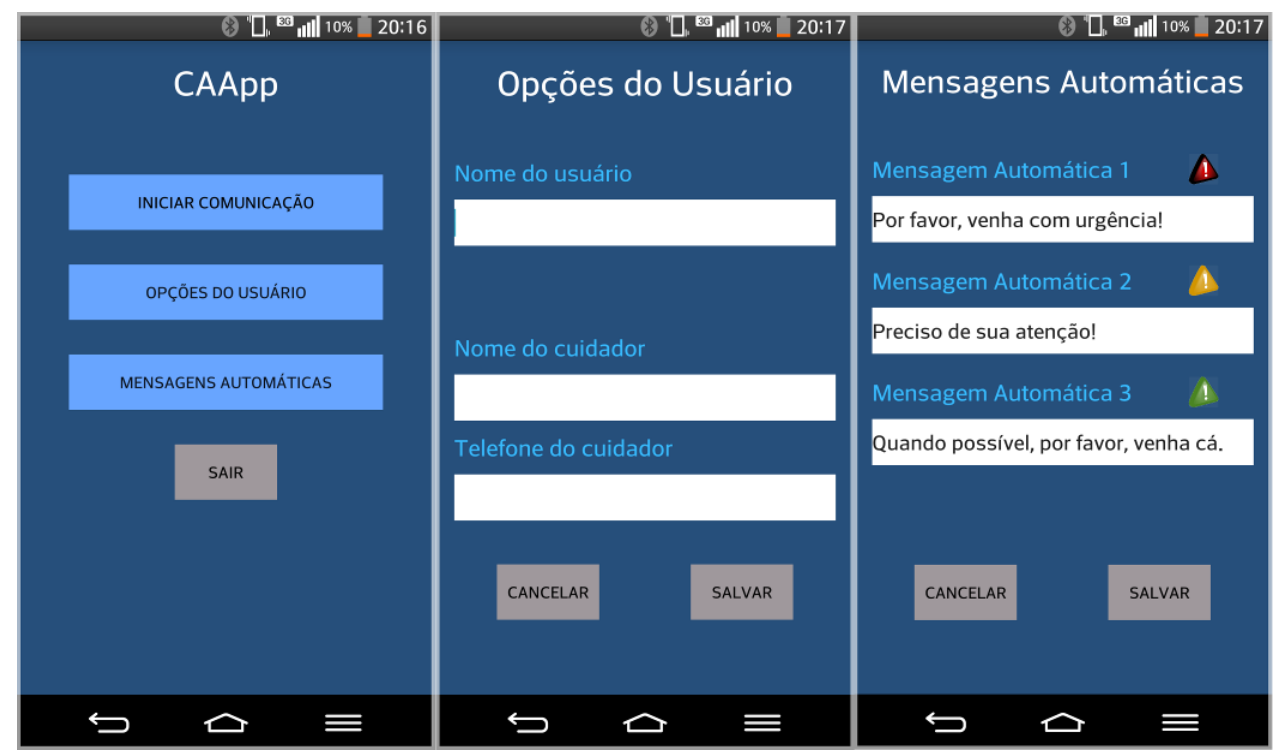

Figura 4. Telas do aplicativo CAApp: a tela inicial (à esquerda), a tela de opções do usuário (no centro) e a tela de mensagens automáticas (à direita).

Assim que o aplicativo é executado, a tela inicial é exibida com três opções disponíveis, como pode-se observar na tela à esquerda na Figura 4. Ao centro da Figura 4, apresenta-se a tela de opções do usuário com as caixas de texto para o cuidador inserir os dados básicos para operação do sistema. A tela de mensagens automáticas, exibida à direita na Figura 4, mostra as caixas de texto para inserção das mensagens e 
apresenta alguns exemplos de texto que podem ser utilizados. Nota-se que a mensagem está relacionada com o símbolo de exclamação que acompanha a caixa de texto, de acordo com o nível de urgência representado.

Após a configuração completa do aplicativo, o cuidador deve selecionar a opção "iniciar comunicação". Neste momento, o aplicativo estabelece a comunicação entre o smartphone e o módulo Bluetooth HC-05 e exibe uma nova tela, na qual permanece aguardando o recebimento de um comando do usuário. A Figura 5 apresenta esta tela e duas possíveis tarefas realizadas pelo aplicativo.

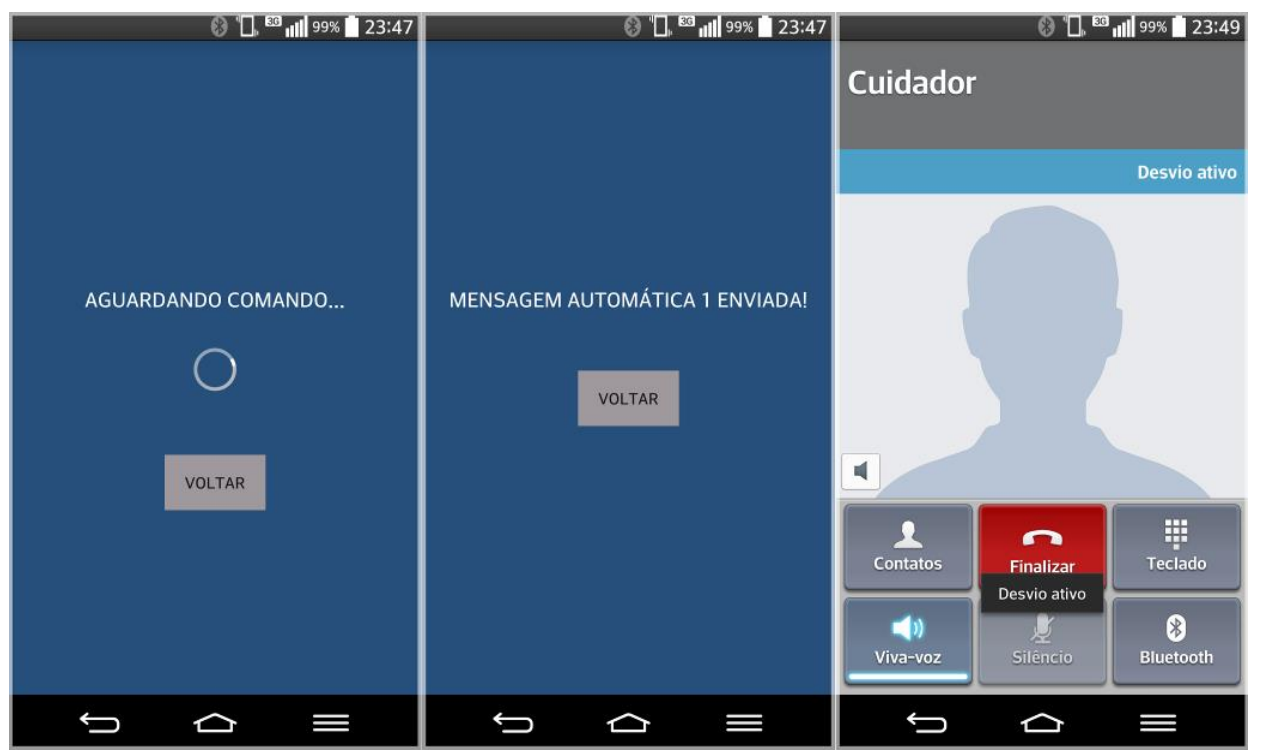

Figura 5. Telas do aplicativo CAApp: aplicativo em operação (à esquerda), confirmação de mensagem enviada (no centro) e ligação iniciada (à direita).

A tela observada à esquerda na Figura 5 aguarda por um comando do usuário. No momento em que o usuário seleciona uma das opções de mensagem de texto na interface gráfica do sistema, o aplicativo recebe o devido comando e o smartphone realiza a tarefa pertinente. A tela apresentada no centro da Figura 5 mostra uma confirmação de mensagem enviada, indicando que o usuário optou por enviar a "mensagem automática 1", no caso. Quando o usuário seleciona a opção de realizar uma ligação telefônica para o cuidador, o smartphone inicia sua tarefa nativa de ligação e ativa o viva-voz, como pode ser observado na tela à direita na Figura 5.

\section{Conclusões}

Este trabalho apresentou o aplicativo CAApp, implementado para ser utilizado em conjunto com o sistema de ambiente inteligente desenvolvido no LAI-UFES. A aplicação desenvolvida neste trabalho validou a proposta de integração de um meio de CAA ao sistema de ambiente inteligente. A partir deste momento, pode-se trabalhar na melhoria do aplicativo e de sua integração com o sistema principal, de forma a oferecer mais recursos de interação e maior liberdade ao usuário. Alguns exemplos de melhorias são: um número maior de contatos disponíveis, a possibilidade do usuário escrever a mensagem de texto e o uso de outros recursos de comunicação do smartphone. 
O aplicativo apresentado deve permanecer sendo executado em tela para permitir o funcionamento do meio de CAA do sistema. É importante destacar que o CAApp foi desenvolvido com este comportamento para oferecer uma melhor visualização da aplicação em operação durante o desenvolvimento deste projeto. No entanto, para não consumir bateria do smartphone sem necessidade, o aplicativo pode ser aprimorado para operar em background, ou seja, em segundo plano, o que será realizado em uma próxima etapa desta pesquisa. Desta forma, o smartphone poderá manter sua tela desligada enquanto não receber um dos possíveis comandos.

\subsection{Trabalhos futuros}

Numa próxima etapa deste trabalho, para validar o sistema de CAA em situação real, pretende-se apresentar o aplicativo CAApp para pessoas com deficiência motora severa e seus respectivos cuidadores. Neste momento, será avaliado o tempo gasto para o usuário realizar cada uma das possíveis operações com o smartphone. Também em um estágio futuro, pretende-se adicionar novas possibilidades de interação com $\mathrm{o}$ smartphone por meio do Emotiv EPOC e da interface gráfica, utilizando diferentes recursos do smartphone. Outra frente de trabalho será o desenvolvimento de uma plataforma na qual o usuário poderá interagir diretamente com um smartphone ou um tablet por meio de sinais biológicos, sem a necessidade do computador de bordo da cadeira de rodas.

\section{Agradecimentos}

Os autores agradecem à Universidade Federal do Espírito Santo (UFES) pela estrutura de laboratórios e equipamentos oferecidos, ao Conselho Nacional de Desenvolvimento Científico e Tecnológico $(\mathrm{CNPq})$ e à Coordenação de Aperfeiçoamento de Pessoal de Nível Superior (CAPES) pelo apoio financeiro e pelas bolsas concedidas.

\section{Referências}

Al-Abdullatif, A., Al-Negheimish, H., Al-Mofeez, L., Al-Khalifa, Nuha (2013) MindControlled Augmentative and Alternative Communication for People with Severe Motor Disabilities. In IEEE $9^{\text {th }}$ Int. Conf. on Innovations in Information Technology, p. 107-112.

Banerjee, A., Chakraborty, S., Das, P., Datta, S., Konar, A., Tibarewala, D. N. e Janarthanan, R. (2012) Single Channel Electrooculogram (EOG) Based Interface for Mobility Aind. In IEEE $4^{\text {th }}$ Int. Conf. on Intelligent Human Computer Interaction, p. $1-6$.

Bissoli, A. L. C., Ferrara, F., Silveira, M. L. e Bastos Filho, T. F. (2015a) Interação com o Ambiente e Comando de Equipamentos por Infravermelho para Cadeirantes. In $1^{s t}$ International Workshop on Assistive Technology, p. 177-180.

Bissoli, A. L. C., Ferrara, F., Sime, M. M. e Bastos Filho, T. F. (2015b) A Multimodal Assistive System to Operate a Smart Environment. In $1^{\text {st }}$ International Workshop on Assistive Technology, p. 103-106.

Blankerts, B., Dornhege, G., Krauledat, M., Schröder, M., Williamson, J., MurraySmith, R. e Müller, K. (2006) The Berlin Brain-Computer Interface Presents the 
Novel Mental Typewriter Hex-o-Spell. In Proceedings of $3^{\text {rd }}$ International Brain Computer Interface Workshop Training Couser, p. 108-109.

Bryen, D. N. e Pecunas P. (2004) Augmentative and Alternative Communication and Cell Phone Use: One Off-the-shelf Solution and Some Policy Considerations. In Assistive technology, p. 11-17. PubMed.

Buning, M. E. (2013) “Adaptações de Alta Tecnologia para Compensar a Incapacidade”, Terapia Ocupacional para Disfunções Físicas, M. V. Radomski e C. A. T. Latham, Brasil, Editora Santos, p. 510-541.

Choi, C., Rim, B. e Kim, J. (2011) Development and Evaluation of a Assistive Computer Interface by sEMG for Individuals with Spinal Cord Injuries. In IEEE International Conference on Rehabilitation Robotics, p. 1-5.

Fager, S., Beukelman, D. R., Fried-Oken, M., Jakobs, T. e Baker, J. (2012) Access Interface Strategies. In Assistive Technology, vol. 24, p. 25-33. Spring.

Fazel-Rezai, R., Allison, B. Z., Guger, C., Sellers, E. W., Kleih, S. C. e Kübler, A. (2012) P300 Brain Computer Interface: Currente Challenges and Emerging Trends. In Frontiers Neuroengineering, vol. 5, article 14, p. 1-14. Frontiers.

Glennen, S. L. (1997) "Introduction to Augmentative and Alternative Communication", Handbook of Augmentative and Alternative Communication, S. L. Glennen e D. C. Decoste, Estados Unidos, Singular Publishing Group Inc, p. 3-20.

Kim, J., Park, H. e Ghovanloo, M. (2012) Tongue-Operated Assistive Technology with Access to Common Smartphone Applications via Bluetooth Link. In IEEE $34^{\text {th }}$ Annual Int. Conference of the Eng. in Medicine and Biology Society, p. 4054-5057.

Lam, Y. M. Non-Acoustic Communication with Speech Smoothing (2012) In Signal \& Imagem Processing: An International Journal, vol. 3, number 1, p. 1-17.

Palerm, C. C. R. e Ruiz, P. A. L. (1992) Augmentative/Alternative System for Public Communication for Children with Cerebral Palsy. In IEEE $14^{\text {th }}$ Annual International Conference of the Engineering in Medicine and Biology Society, p. 1525-1526.

Paul, G. M., Cao F., Torah, R., Yang K., Beeby, S. e Tudor, J. (2014). A Smart Textile Based Facial EMG and EOG Computer Interface. In IEEE Sensors Journal, vol. 14, issue 2, p. 393-400.

Pedretti, L. W. e Early, M. E. (2004) "Desempenho Ocupacional e Modelos de Prática para Disfunção Física”, Terapia Ocupacional: Capacidades Práticas para Disfunções Física, capítulo 1.

Quintela, M. A., Mendes, M. e Correia, S. (2013) Augmentative and Alternative Communication: Vox4all ${ }^{\circledR}$ Presentation. In IEEE $8^{\text {th }}$ Iberian Conference on Information Systems and Technologies, p. 1-6.

Torii I., Ohtani K., Niwa T., Yamamoto A. e Ishii N. (2012) Augmentative and Alternative Communication with Digital Assistant for Autistic Children. In IEEE International Conference on Emerging Signal Processing Applications, p. 71-74.

USSAAC - United States Society for Augmentative and Alternative Communication (2015) “AAC Devices”, disponível em: http://www.ussaac.org/aac-devices.cfm 